\title{
Recent Ultra High Energy neutrino bounds and multimessenger observations with the Pierre Auger Observatory
}

\author{
Enrique $Z^{1, \star}{ }^{1, \star}$ for The Pierre Auger Collaboration ${ }^{2, \star \star}$ \\ ${ }^{1}$ Depto. Física de Partículas \& Instituto Galego de Física de Altas Enerxías, Universidade de Santiago de \\ Compostela, 15782 Santiago de Compostela, Spain \\ ${ }^{2}$ Observatorio Pierre Auger, Av. San Martín Norte 304, 5613 Malargüe, Argentina
}

\begin{abstract}
The overall picture of the highest energy particles produced in the Universe is changing because of measurements made with the Pierre Auger Observatory. Composition studies of cosmic rays point towards an unexpected mixed composition of intermediate mass nuclei, more isotropic than anticipated, which is reshaping the future of the field and underlining the priority to understand composition at the highest energies. The Observatory is competitive in the search for neutrinos of all flavors above about 100 $\mathrm{PeV}$ by looking for very inclined showers produced deep in the atmosphere by neutrinos interacting either in the atmosphere or in the Earth's crust. It covers a large field of view between $-85^{\circ}$ and $60^{\circ}$ declination in equatorial coordinates. Neutrinos are expected because of the existence of ultra high energy cosmic rays. They provide valuable complementary information, their fluxes being sensitive to the primary cosmic ray masses and their directions reflecting the source positions. We report the results of the neutrino search providing competitive bounds to neutrino production and strong constraints to a number of production models including cosmogenic neutrinos due to ultra high energy protons. We also report on two recent contributions of the Observatory to multimessenger studies by searching for correlations of neutrinos both with cosmic rays and with gravitational waves. The correlations of the directions of the highest energy astrophysical neutrinos discovered with IceCube with the highest energy cosmic rays detected with the Auger Observatory and the Telescope Array revealed an excess that is not statistically significant and is being monitored. The targeted search for neutrinos correlated with the discovery of the gravitational wave events GW150914 and GW151226 with advanced LIGO has led to the first bounds on the energy emitted by black hole mergers in Ultra-High Energy Neutrinos.
\end{abstract}

\section{Introduction}

Cosmic Rays (CR) are energetic protons and nuclei that reach the Earth giving a wealth of information about the Universe around us. Galactic CR are believed to be produced by Fermi acceleration in Supernovae shocks. Ultra-High Energy CR (UHECR) reach astonishing large energies above $10 \mathrm{EeV}$ $\left(1 \mathrm{EeV}=10^{18} \mathrm{eV}\right)$ producing interactions with the atmospheric nuclei with center of mass energies well

\footnotetext{
${ }^{\star}$ e-mail: enrique.zas@usc.es

$\star \star$ e-mail: auger_spokespersons@fnal.gov
} 
exceeding those reached in accelerator experiments. They can travel intergalactic distances and give information on the sources where they are produced and on the interactions they suffer as they travel to Earth. For instance, the most energetic particles are believed to be little deviated by the galactic and intergalactic magnetic fields and their direction could help to unveil the highly uncertain strength and pattern of these magnetic fields. Extracting information from UHECR is however an extremely challenging task because we do not yet know the sources of UHECR, we have limited information about the primary mass composition (and electric charge) [1] and, in addition, the models we have to describe their interactions are not sufficiently accurate [2].

High energy cosmic rays induce Extensive Air Showers (EAS) in the atmosphere as ultrarelativistic particles are produced in successive colisions with air molecules. Above EeV energies they reach shower maximum containing over $10^{10}$ particles that spread over several $\mathrm{km}$ of radius after traversing a depth of order $800 \mathrm{~g} \mathrm{~cm}^{-2}$. The Pierre Auger Observatory consists of a $3000 \mathrm{~km}^{2}$ array of water-Cherenkov detectors (SD) in a hexagonal grid of $1.5 \mathrm{~km}$ spacing which is used to detect the particle front of EAS and a Fluorescence Detector (FD) that monitors the emissions form excited Nitrogen atoms as the shower develops over this surface. Located in Mendoza, Argentina, at an average latitude of $35.2^{\circ} \mathrm{S}$ and at a mean atmospheric depth (altitude) of $875 \mathrm{~g} \mathrm{~cm}^{-2}$ (1400 m) [10] it has been running since its construction started in 2004. The FD gives a calorimetric measurement of the energy deposition in the atmosphere tracking the shower development which combined with the SD area gives the largest and most precise detector ever built to measure EeV cosmic rays. ${ }^{1}$

The spectrum measurements made at the Observatory have revealed a supression of the flux at energies above $40 \mathrm{EeV} \mathrm{[4]} \mathrm{which} \mathrm{cannot} \mathrm{be} \mathrm{unambiguosly} \mathrm{attributed} \mathrm{to} \mathrm{photoproduction} \mathrm{with} \mathrm{the}$ Cosmic Microwave Background (CMB), the Greisen-Zatsepin-Kuz'min cut-off, because composition measurements indicate a transition to increasing mass primaries as the energy increases above about $2 \mathrm{EeV} \mathrm{[1]} \mathrm{which} \mathrm{is} \mathrm{also} \mathrm{suggestive} \mathrm{of} \mathrm{an} \mathrm{intrinsic} \mathrm{maximum} \mathrm{acceleration} \mathrm{energy} \mathrm{in} \mathrm{the} \mathrm{sources}$ which is proportional to the nucleus charge, $Z$. Such findings are taking long to permeate the community, strongly predjudiced in favor of a pure proton composition as supported by TA Collaboration claims [5]. A joint analysis has revealed that TA and Auger data are $X_{\max }$-consistent: while Auger data are not compatible with pure protons at $10 \mathrm{EeV}$ in any of the available hadronic models, the TA data, having larger uncertainties, are compatible with both protons and Auger composition results [6].

The measured arrival directions of UHECR are shedding light into the complex picture. A dipole anisotropy of $6.5 \%$ for energies above $8 \mathrm{EeV}$ was discovered with $5.2 \sigma$ significance pointing to $(l, b)=$ $\left(233^{\circ},-13^{\circ}\right)$ in galactic coordinates, $125^{\circ}$ away from Galactic Center [7]. Evidence for extragalactic sources is now compelling as it is reasonably aligned to the excess dipole in the galaxy distribution [7]. Interestingly a $3.9 \sigma$ significance has also been reported for correlations of arrival directions with the locations of Star Burst Galaxies (SBG) at angular scales $\sim 10^{\circ}$ and CR energies above $40 \mathrm{EeV}$ [8]. At these energies composition inference is lacking because of the limited duty cycle of the FD. Clearly measurements are closing in on the UHCER sources, but more data are needed.

Neutrinos provide complementary information. Interactions of UHECR in the sources or during propagation produce $\mathrm{EeV}$ neutrinos such as the cosmological neutrinos resulting from their GZKinteractions with the CMB. Having no charge, neutrinos point to their production sites even when they are shielded by large amounts of matter. Their energy spectrum is also related to cosmic ray composition at the highest energies, which is largely unknown. As extraterrestrial neutrinos of several $\mathrm{PeV}$ have already been detected [9], extending the spectrum measurement to the EeV region is most natural to effectively constrain the production mechanisms of UHECR. Their detection would probe neutrino interactions at energies orders of magnitude above accelerator experiments. Information about flavor ratios besides constraining the production mechanisms may even signal new physics.

\footnotetext{
${ }^{1}$ Note that the Telescope Array (TA) [3] is about a fourth of the size of the Observatory and has been running for less time.
} 

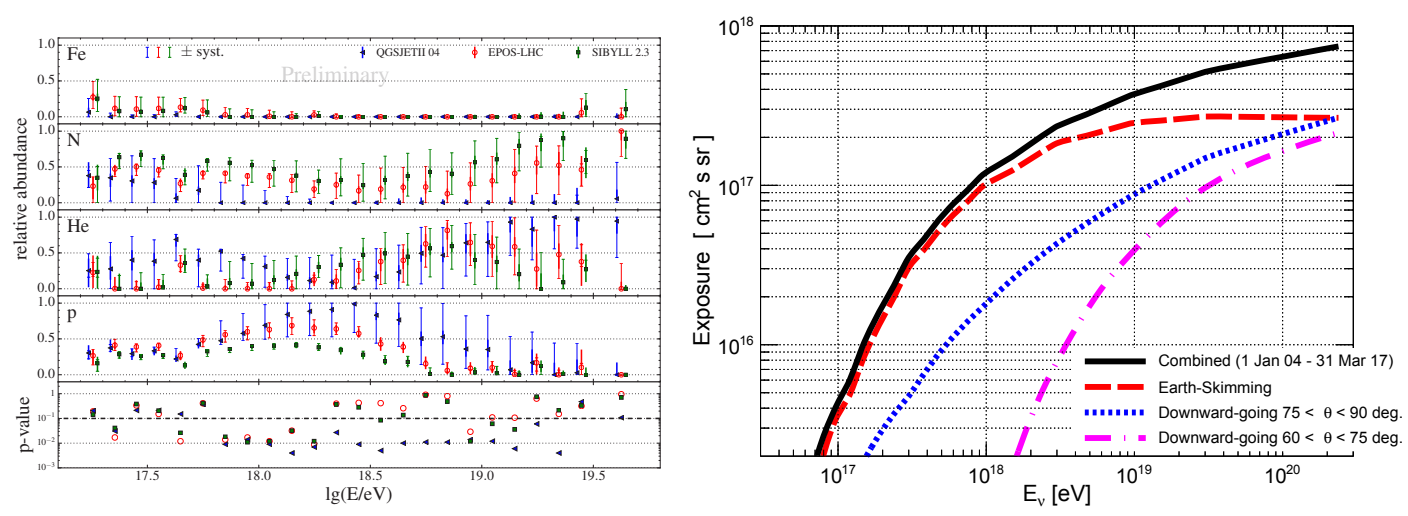

Figure 1. Left: Composition measured at the Pierre Auger Observatory using $X_{\max }$ as a function of energy. Right: Total exposure (solid) as a function of neutrino energy and contributions of the ES (dashes), DGH (dots) and DGL (dot dashes) searches at Earth assuming equal fluxes for all neutrino flavors.

\section{Neutrino bounds with the Pierre Auger Observatory}

It has been known for long that the Pierre Auger Observatory can also be used to detect inclined neutrino interactions deep in the atmosphere [11] and nearly horizontal Earth-skimming (ES) tau neutrinos [12]. If an ES tau neutrino has a charged current interaction near the surface of the Earth, the tau lepton will often exit to the atmosphere and decay in flight, producing a nearly horizontal EAS. This channel is only sensitive to tau neutrinos mostly between $\sim 0.1$ and $25 \mathrm{EeV}$, where cosmological neutrinos are expected. Deep inclined showers produced by down-going (DG) neutrinos and by ES tau-neutrinos can be distinguished from showers due to cosmic rays that interact in the upper layers because their electromagnetic component reaches the particle detectors. In contrast, the electromagnetic component of inclined cosmic ray showers gets absorbed after $\sim 800 \mathrm{~g} \mathrm{~cm}^{-2}$ (shower maximum) and only the muon component reaches ground level. These showers, which are regularly detected and reconstructed [13], consist of $\sim 20$ to $200 \mathrm{GeV}$ energy muons that travel nearly parallel to the shower axis and undergo few interactions before reaching the ground. As a result they accumulate small time delays and deposit the energy in a short burst, while deep neutrino showers produce signals with a much broader time spread because of the abundance of electrons and photons.

The time structure provides the basis for neutrino identification [11]. The signal of the SD stations of the Observatory is digitized in $25 \mathrm{~ns}$ time bins allowing a detailed measurement of the time profile of the shower front. Extensive simulations of neutrino events have been made varying the energy, zenith and azimuth angles, depth of interaction and neutrino impact parameter and, in the ES case, the tau lepton decay altitude. The response of the SD is also simulated with standard software. The search strategy involves a pre-selection of inclined events followed by a selection of neutrino candidates. An optimized variable is chosen, studying signal and background distributions after applying the same selection. A cut is made to separate simulated neutrinos from the background of cosmic showers.

The selection process is optimized splitting it in three categories: (1) ES selection requires at least three stations, a high eccentricity of the triggered area on the ground, an apparent average speed of the signal close to $c$ and a small spread. The selection of DG neutrinos requires at least 4 stations and is further split in High (DGH) and Low (DGL) zenith angle ranges. (2) DGH selection for zenith angles 

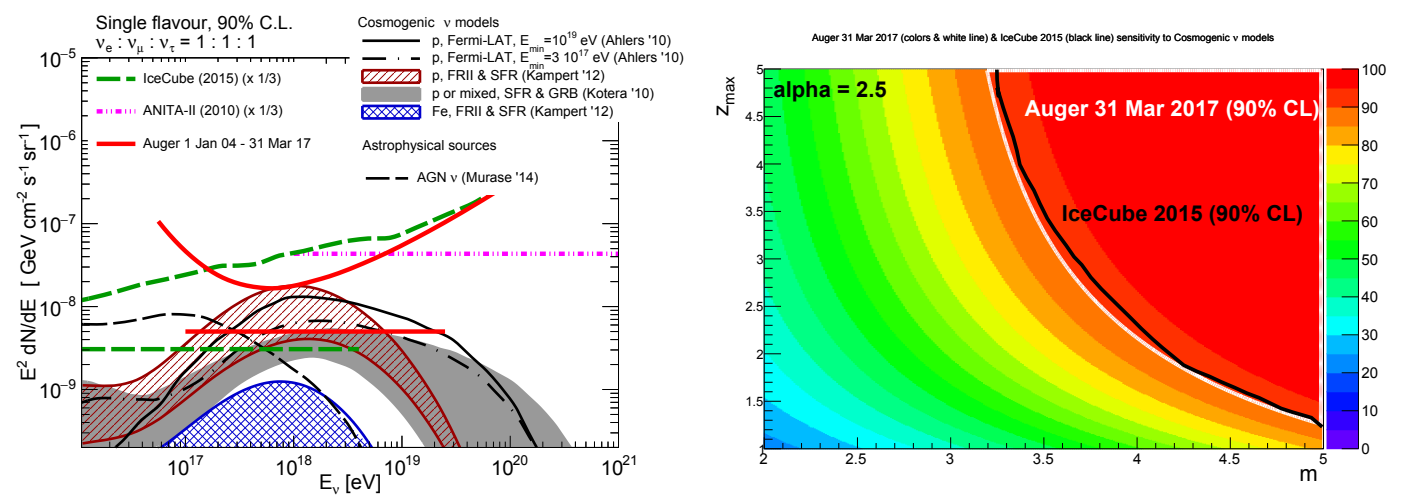

Figure 2. Left: Integral upper limit (at $90 \%$ C.L.) for the normalization, $k$, of a diffuse flux $d N / d E_{v}=k E^{-2}$ of single flavor neutrinos (straight red line), and differential upper limit (see text) compared to those from ANITAII [15] and IceCube [16] and several predictions (cosmogenic [17-19], astrophysical [20].) All flavors are assumed to have equal fluxes. Right: Constraints on parameter space for cosmological neutrinos in proton models $\left(d N / d E \propto E^{-2.5}\right)$ as a function of $m$ and $z_{\max }$ [29] (see text). Colored areas represent levels of exclusion. The region above the black (white) line is excluded at 90\% C.L. by IceCube [16] (Auger) data.

exceeding $75^{\circ}$ after a plane fit reconstruction must satisfy less stringent cuts on the same ES variables. (3) DGL selection for zenith angles in the $58.5^{\circ}$ to $76.5^{\circ}$ range [14].

The discriminating variable is related to the width of the digitized signal trace namely the Area over Peak (AoP). For the ES selection it is the average AoP of the triggered stations $(\langle\mathrm{AoP}\rangle)[14]$ and for the DG cases up to ten variables related to the AoP of 4 or 5 early or central stations are combined in a Fisher discriminant approach [14]. The cut value is chosen using a small fraction of the data which is assumed to be made up of cosmic ray showers and demanding a background rate of one event every 50 years. No neutrino candidates have been found.

The effective exposure as a function of neutrino energy convolved with a given neutrino flux spectrum, gives the expected number of events. It is obtained as the integral of the interaction and detection (trigger, selection and identification) probabilities over surface area, solid angle and time. For ES tau-neutrinos the probability of the tau-lepton exiting the Earth is calculated using a code that includes regeneration. It is then multiplied the probabilities of the tau decaying (a standard exponential) and by the detection probability. Both depend on decay position and this variable must be integrated too. For the DG selection the neutrino flavor and the type of interaction must be considered carefully to calculate interaction and detection probabilities. The time integral is performed sampling the effective probabilities over array configurations chosen every three days. The searches presented here span data collected from 1 January 2004 to 31 March 2017. Results are displayed in Fig. 1-right, assuming all flavors have equal fluxes. The ES channel dominates below $\sim 50 \mathrm{EeV}$.

The $90 \%$ C.L. limits for a diffuse isotropic flux of single flavor neutrinos are shown in Fig. 2, compared to results from other telescopes. A more restrictive bound is obtained integrating a conventional $k E^{-2}$ neutrino spectrum, giving $k<5 \times 10^{-9} \mathrm{GeVcm}^{-2} \mathrm{~s}^{-1}$, and plotted between 0.1 to $25 \mathrm{EeV}$, where $90 \%$ of the events are expected. The differential bound, obtained integrating in bins of 0.5 in $\log _{10} E_{\nu}$, illustrates the good SD sensitivity for detecting EeV neutrinos. Several predictions for cosmogenic neutrinos from models that address the UHECR origin are excluded at the $90 \%$ C.L., particularly those assuming proton primaries and attributing strong redshift evolution to the sources $[18,19]$. Exclusion plots can be made as function of relevant parameters, namely the source evolution $(m)$, the maximum 

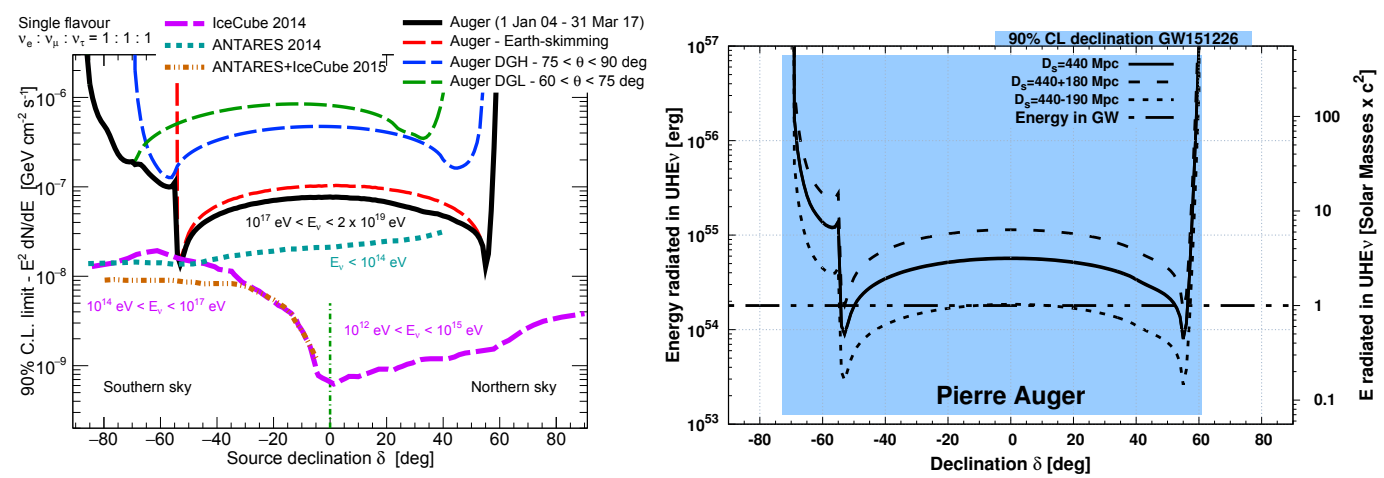

Figure 3. Left: Upper limits at $90 \%$ C.L. for a single flavor neutrino flux $d N / d E_{v}=k^{\mathrm{PS}} E_{v}^{-2}$ as a function of $\delta$ for ES, DGH and DGL searches as labeled, compared to those of for IceCube [22], ANTARES [23] and a combination of both [24] (equal flavor scenario). Right: Constraints on the radiated energy in UHE neutrinos (per flavor) from the source of GW151226 as a function of $\delta$ [26]. Energies above the solid line are excluded at the $90 \%$ C.L.. The calculation is done for a distance $D_{s}=410 \mathrm{Mpc}$ (dashed lines correspond to reported distances at the $90 \%$ C.L.). The inferred energy radiated in gravitational waves, $E_{\mathrm{GW}} \simeq 1.9 \times 10^{54} \mathrm{erg}$ [27], is also displayed (dot-dashed line ). The shaded region indicates the $90 \%$ C.L. region for the event location.

redshift $\left(z_{\max }\right)$, the spectral index $(\alpha)$ and the maximum energy of the UHECR flux $\left(E_{\max }\right)$ [28], using a conservative analytical approximation of the cosmogenic neutrino flux [29]. An example for a spectral index of 2.5 and $E_{\max }=300 \mathrm{EeV}$ is shown in Fig. 3.

\section{Point source searches and correlations}

Bounds on point sources as a function of declination $(\delta)$, obtained integrating the exposure in right ascension, are displayed in Fig. 3 [21]. The ES exposure displays strong peaks because this channel is very effective at converting the tau neutrinos into exiting tau leptons for directions close to the horizontal. The bulk of the ES sensistivity is for zenith angles between $90^{\circ}$ and $92.5^{\circ}$ and the sky coverage is $-54.5^{\circ}<\delta<59.5^{\circ}$. The DG search extends it South to $-84.5^{\circ}$.

The discovery of gravitational wave events reported by the Advanced Ligo Collaboration has triggered an intense program of targeted searches for coincidence events. A search has been made for $\mathrm{EeV}$ neutrinos in the Observatory data correlated with black-hole binary coalescences GW150914, GW151226, GW170104 and LVT151012 (candidate) [27]. Assuming a steady flux during a day after the event, motivated by an upper limit to the duration of GRB afterglows [26], a bound on the EeV neutrino emission has been obtained. The directionality of the neutrino exposure makes the search dependent on event position (only known as a likelihood plot over a broad region) and the results are given in terms of declination $(\delta)$. The neutrino exposure averaged over a day covers a large fraction of the sky and has thus large overlap with all the events, as exemplified by the shadowed band in Fig. 3 for event GW151226, for which practically the whole $90 \%$ C.L. declination band is covered. The $90 \%$ C.L. bound, obtained for an $E^{-2}$ spectrum and converted into a bound on the energy fluence radiated in EeV neutrinos, is shown in Fig. 3 for GW151226. Similar bounds for GW150914 are somewhat stronger while for GW170104 they are weaker because it is further away [26].

Correlations between directions of UHECR detected with the Auger Observatory and TA and those of the neutrinos detected with IceCube have also been searched for. Angular correlations between neutrino and UHECR pairs have been compared to expectations from isotropy (as a function of 
separation angle). A stacking method (fixing both the neutrino and the UHECT directions) has been also used to maximize the likelihood varying the fraction of correlating events. Plausible assumptions about angular dispersion are required to account for direction tolerances. First reports pointed to interesting correlations [30] that have nevertheless become weaker in a recent update of the analysis [31].

Searches for anisotropies of UHECR are revealing precious information about their production sites but more data are needed. The Auger Observatory is effective to constrain models searching for diffuse neutrino fluxes and for point sources as part of a multi-messenger approach. As data from the Observatory continue to be analyzed and combined with other experiments we will get closer to deciphering the elusive origin of the most energetic particles in the Universe.

\section{References}

[1] A. Aab et al. Phys. Rev. D 90, 122005 (2014).

[2] E. Zas J. Phys. Conf. Ser. 632, no. 1, 012104 (2015).

[3] Abu-Zayyad, T. et al.; Nucl.Instrum.Meth. A 689 (2013) 87-97.

[4] J. Abraham et al. Phys. Rev. Lett. 101, 061101 (2008).

[5] R. U. Abbasi et al., Astropart. Phys. 64, 49 (2015).

[6] V. de Souza for the Pierre Auger and TA Collaborations; Proc. of ICRC 2017, PoS (2017) 522.

[7] A. Aab et al. (in press) Science 358, (2017).

[8] G.U. Giaccari for the Pierre Auger Collaboration; Proc. of ICRC 2017, PoS (2017) 483.

[9] M. G. Aartsen et al. Phys. Rev. Lett. 113, 101101 (2014).

[10] A. Aab et al. Nuc. Inst. Meth. A 789, 172 (2015).

[11] K. S. Capelle et al.; Astropart. Phys. 8, 321 (1998).

[12] X. Bertou et al.; Astropart. Phys. 17, 183 (2002).

[13] A. Aab et al.; JCAP 1408, 019 (2014).

[14] A. Aab et al. [Pierre Auger Collab.], Phys. Rev. D 91, 092008 (2015).

[15] P. W. Gorham et al. [ANITA Collab.], Phys. Rev. D 85, 049901(E) (2012).

[16] M. G. Aartsen et al. [IceCube Collab.] Phys. Rev. Lett. 117, 241101 (2016).

[17] M. Ahlers et al., Astropart. Phys. 34, 106 (2010).

[18] K. -H. Kampert, M. Unger, Astropart. Phys. 35, 660 (2012).

[19] D. Allard et al., JCAP 10, 013 (2010).

[20] K. Murase, Y. Inoue, and C. D. Dermer, Phys. Rev. D 90, 023007 (2014).

[21] P. Abreu et al., Astrophys. J. 755, L4 (2012).

[22] M.G. Aarsten et al., Astrophys. J. 796, 109 (2014).

[23] S. Adrian-Martinez et al. [ANTARES Collab.], Astrophys. J. Lett. 786, L5 (2014).

[24] S. Adrian-Martinez et al., Astrophys. J. 823, 65 (2016).

[25] K. Kotera and J. Silk; Astrophys. J. 823, no. 2, L29 (2016).

[26] A. Aab et al. [Pierre Auger Collab.], Phys. Rev. D 94, 122007 (2016).

[27] B. P. Abbott et al. [LIGO Scientific Collab. and Virgo Collab.], Phys. Rev. Lett. 116, 061102 (2016); ibid. 116241103 (2016); ibid. 118221101 (2017).

[28] M. G. Aartsen et al. Phys. Rev. Lett. 117, 241101 (2016).

[29] S. Yoshida and A. Ishihara, Phys. Rev. D 85, 063002 (2012).

[30] M.G. Aartsen et al.; JCAP 1601, 037 (2016).

[31] I. Al Samarai and G. Golup; Proc. of ICRC 2017, PoS (2017) 961. 